\title{
DETERMINATION OF ATOMISTIC STRUCTURE OF Ni-BASE SINGLE CRYSTAL SUPERALLOYS USING MONTE CARLO SIMULATIONS AND ATOM-PROBE MICROANALYSES
}

\author{
H. Murakami, Y. Saito* and H. Harada \\ National Research Institute for Metals, Computational Materials Science Division, \\ 1-2-1, Sengen, Tsukuba Science City, 305, Japan \\ *Department of Materials Science and Engineering, School of Science and Engineering, \\ Waseda University, 3-4-1, Okubo, Shinjuku-ku, Tokyo 169, Japan.
}

\section{$\underline{\text { Abstract }}$}

The atomic locations of alloying elements in some Ni-base single crystal superalloys have been investigated using Monte Carlo Simulations (MCS), and the predictions have been compared with the experimental results obtained from atom-probe field ion microscopy (APFIM). The $\gamma$ and $\gamma$ phase compositions and the site occupancy of alloying elements in the $\gamma^{\prime}$ phase at equilibrium conditions were predicted using MCS. The predictions were then compared with estimates obtained by the Cluster Variation Method (CVM) and experimental results obtained by APFIM, so as to verify the applicahility of MCS. It was found that the MCS estimations were generally in good agreement with both CVM predictions and APFIM results. For multicomponent systems, alloying elements may be classified into three general groups: (1) those which preferentially partition to the $\gamma$ phase and substitute for the Al site in the $\gamma^{\prime}$ phase, such as $\mathrm{Ti}$ and $\mathrm{Ta}$; (2) those which preferentially partition to the $\gamma$ phase and substitute for the Ni site in the $\gamma^{\prime}$ phase, such as $\mathrm{CO}$; (3) those which generally partition to the $\gamma$ phase and substitute for the $\mathrm{Al}$ site in the $\gamma^{\prime}$ phase, such as Re, Mo and $W$. In this paper, virtual experiments were also performed to investigate the chemistry of $\gamma / \gamma^{\prime}$ interfaces.

\section{Introduction}

Although nickel-base single crystat superalloys have already been developed extensively for turbine blade applications, there are continuing efforts to improve the mechanical properties of these alloys for next-generation aeroengines. The superalloys have $\mathrm{Ll}_{2}$ ordered $\gamma^{\prime}$ precipitates in $\gamma$ matrices. Their mechanical properties are thus expected to be controlled by the microstructural parameters of the two phases, such as their inherent mechanical properties, and the interaction between the two phases. It is therefore of the utmost importance to investigate the atomic configurations of alloying clements which accordingly determine the characteristics of the two phases and the mechanical properties.

The Cluster Variation Method (CVM) has been applied to predict the atomic configurations of alloying elements in nickel-base superalloys ${ }^{(1.2)}$. Using the CVM, the atomic location of solute atoms

$$
\text { Superalioys } 1996
$$

in both phases at equilibrium is estimated as a function of alloy composition and temperature.

The interaction between the $\gamma$ and $\gamma^{\prime}$ phases also affects the mechanical propertics of supcralloys. It is thus important to understand the chemistry of $\gamma / \gamma^{\prime}$ interfaces, where atomic configurations are not expected to reach the equilibrium state. Monte Carlo Simulations (MCS)(3) have been employed to obtain such interface information. This method enables predictions of temporal and spatial evolution of the atomic arrangement, and the kinetics of ordering of nickel-base superalloys. An additional advantage of MCS is that, since the position of every single atom in the system is determined, three dimensional representation of the atomic configurations is possible. Therefore, the distinction between ordered and disordered regions, and the chemistry at interfaces may be visualised.

However, the theoretical models will not be of practical use until the agreement between predictions and experimental results is demonstrated. In order to verify theoretical estimates obtained from MCS and CVM, experinental analysis using Alom-Probe Field Ion Microscopy (APFIM) has been carried out. APFIM, which has subnanometer spatial resolution, is a powerful tool for experimentally obtaining atomic information of materials. The main objective of this study is to understand the atomic configurations of some multicomponent nickel-base superalloys by comparing the predictions of MCS with experimentally obtained APFIM results, so that a novel alloy design program, which will predict not just the atomic structure but also estimate the mechanical properties of these superalloys, may be established in the near future.

In this paper, the atomic configurations at equilibrium conditions, such as the $\gamma$ and $\gamma^{\prime}$ phase compositions and the site occupancy behaviour of alloying elements in the $\gamma^{\prime}$ phase, determined by MCS, CVM and APFIM in five multicomponent nickel-base single crystal superalloys under equilibrium conditions, are compared so as to verify the applicability of MCS and CVM. Additionally, the chemistry at the $\gamma / \gamma^{\prime}$ interface simulated by MCS is compared with that obtained by APFIM. 
TABLE 1 COMPOSITION OF ALLOYS (in at. \%)

\begin{tabular}{c|cccccccccc}
\hline ALLOY & $\mathrm{Ni}$ & $\mathrm{Al}$ & $\mathrm{Ti}$ & $\mathrm{Cr}$ & $\mathrm{Co}$ & $\mathrm{Mo}$ & $\mathrm{Hf}$ & $\mathrm{Ta}$ & $W$ & $\mathrm{Re}$ \\
\hline CMSX2 & 67.4 & 12.5 & 1.2 & 9 & 5 & 0.4 & - & 2 & 2.5 & - \\
CMSX4 & 63.0 & 12.6 & 1.3 & 7.6 & 9.8 & 0.4 & 0.03 & 2.2 & 2.1 & 1.0 \\
MC2 & 65.9 & 11.2 & 2.5 & 9.3 & 5.1 & 1.3 & - & 2.0 & 2.6 & - \\
TMS-63 & 72.0 & 12.8 & - & 7.8 & - & 4.6 & - & 2.8 & - & - \\
TMS-71 & 66.6 & 12.7 & - & 6.9 & 6.2 & 4.0 & - & 2.8 & - & 0.8 \\
\hline
\end{tabular}

Experimental

The materials investigated in this study are listed in Table 1. Among these alloys, first generation $\mathrm{CMSX} 2^{(4)}$ and second generation CMSX ${ }^{(5)}$, provided as a $20 \mathrm{~mm}$ diameter bar provided by Rolls Royce plc, are in commercial use. TMS-63(6,7) and TMS-71(8) were developed by the National Research Institute for Metals (NRIM) employing the regression analysis based Alloy Design Program (ADP) ${ }^{(6,7,9)}$. Details of thermal treatment, sample preparation and the experimental results for alloys CMSX2, CMSX4, MC2, TMS-63 and TMS-71 have been described in previous papers $(8,10-13)$. Note that for all the alloys, the final ageing treatment was conducted at about $1313 \mathrm{~K}$. The temperature of the system used for both CVM and MCS was thus set to $1313 \mathrm{~K}$.

\section{Procedure of Numerical Simulations}

\section{Energy calculation}

Both the CVM and MCS require appropriate modelling of atomic interactions in order to calculate total energy change in the systems. Lennard-Jones pair potentials were employed in order to simplify the numerical simulations. The Lennard-Jones pair potentials $e_{i j}(r)$ are described by:

$$
e_{i j}(r)=e_{i j}^{0}\left[\left(r_{i j} / r\right)^{m_{i j}}-\left(m_{i j} / n_{i j}\right) /\left(r_{i j} / r\right)^{n_{i j}}\right]
$$

where $r_{i j}$ is the interatomic distance between atomic species $i$ and $j$ for which $e_{i j}(r)$ reaches is minimum value i.e.. $e_{i j}(r)=-e_{i j}^{0}$.

It has been shown by Sanchez et al.(14) that the best values for the exponents $n_{i j}, m_{i j}$ of the attractive and repulsive potentials of metals are 4 and 8 , respectively. Details of the method for determining pair potential parameters and the procedure for the CVM are described in previous papers $(1.2)$

\section{Algorithm for MCS data acquisition}

For MCS, the initial structure may be generated by randomly assigning atoms to lattice sites. The kinetics of ordering may be controlled by the direct exchange of a randomly selected atom with one of its neighbouring atoms (Kawasaki dynamics(15)). The probability $W$ that an exchange trial is accepted is given by:

$$
W=\exp (-\Delta H / k T) /[1+\exp (-\Delta H / k T)]
$$

where $\Delta H$ is the change in energy associated with the exchange of the atoms, $k$ is the Boltzmann's constant and $T$ is the absolute temperature. $\Delta H$ is calculated by taking account of contributions from only the first nearest neighbour atoms.

The simulations were performed on a $16 \times 15 \times 16$ unit cell mesh (16,384 atoms) mainly for visualisation, and on a $32 \times 32 \times 32$ unit cell mesh $\left(131,072\right.$ atoms) mainly for determination of the $\gamma$ and $\gamma^{\prime}$ phase compositions and for the investigation of the site occupancy behaviour of alloying elements in the $\gamma^{\prime}$ phase. Periodic boundary conditions were employed. It should be noted that all the lattice positions in the system were fixed: i.e. no relaxation effects were considered in the calculation. A Hewlett Packard Model 712/80 workstation system was used for the calculations. The simulations were carried out until the atomic configurations were regarded as equilibrium (typically 20,000 Monte Carlo steps): the total enthalpy change of the system proved to be negligibly small after 20,000 Monte Carlo steps.

Fig. 1 shows a 3-dimensional representation of the atomic arrangement of solute atoms in TMS-71, obtained by MCS after 20,000 Monte Carlo steps. Here, the 22nd atomic layer of the system is highlighted. The distribution of atoms in the system may be thus described. Fig. 2 shows the 2-dimensional arrangement of atoms on the 22 nd to the 25 th layers, normal to the $<100\rangle$ direction. The atom species in TMS-71 may be identified by their size and brightness. Since pure $\mathrm{Ni}$ layers and $\mathrm{Ni}$-Al mixed layers must appear in turn along $\langle 100\rangle$ for pure fully-ordered $\mathrm{Ni}_{3} \mathrm{Al}$, the atomic locations on the (100) layers in the $\gamma^{\prime}$ phase for multicomponent Ni-base superalloys will be represented as shown in Fig. 3. From MCS, it is thus possible to identify the ordered $\gamma^{\prime}$ phase, as indicated by arrows in Fig. 2, the atomic configurations of which are almost identical to the expected representation (Fig. 3). From Fig. 2, the site occupancy of alloying elements may also be understood.

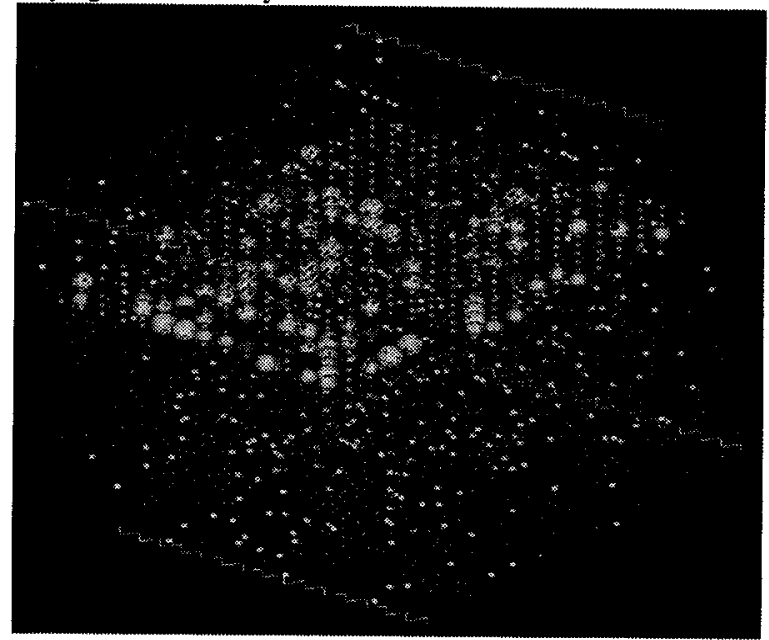

Figure 1. The 3-dimensional representation of the atomic arrangement of solute atoms in TMS-71, obtained by MCS after 20,000 Monte Carlo steps 


\begin{tabular}{|c|c|c|c|c|c|c|c|c|c|c|}
\hline$A 1.2 O Y$ & $\mathrm{Ni}$ & $A i$ & $T_{i}$ & $\mathrm{Cl}$ & $\mathrm{Co}$ & Mo & Hf & $T_{a}$ & $w$ & Re \\
\hline $\mathrm{CMSX} 2$ & 63.4 & 12.5 & 1.2 & 9 & 5 & 0.4 & 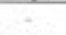 & 2 & 2.5 & - \\
\hline CMSX4 & 63.0 & 12.6 & 1,3 & 7.6 & 9.8 & 0.4 & 0,03 & 2.2 & 2.1 & 1.0 \\
\hline $\mathrm{MC2}$ & 65,9 & 11.2 & 2.5 & 9.3 & 5.1 & 1.3 & & 2.0 & 2.6 & \\
\hline TMS. 63 & 72.0 & 12.8 & - & $7, k$ & & 4,6 & & 2.8 & - & \\
\hline TMS-71 & 66,6 & 12.7 & $=$ & 6.9 & 6,2 & 4.0 & * & 2.8 & - & 0.8 \\
\hline
\end{tabular}

\section{Experimental}

The mutcrials investigated in this study are listed in Table 1. Among these alloys, first generation CMSX2(4) and second generation CMSX4(5), provided as a $20 \mathrm{~mm}$ diameter bar provided by Rolls Royce ple, are in commercial use. TMS-63(6,7) and TMS-7I(8) were developed by the National Research Institute for Metals (NRIM) employing the regression analysis based Alloy Design Program (ADPye .7.91, Details of thermal treatment, sample preparation and the experimental results for alloys CMSX2, CMSX4, MC2, TMS-63 and TMS-71 have been described in previous papers ${ }^{(8,10-13)}$. Note that for all the alloys, the final ageing treatment was conducted at atbout $1313 \mathrm{~K}$. The temperature of the system used for both CVM and MCS was thes set to $1313 \mathrm{~K}$.

\section{Procedure of Numerical Simulations}

\section{Energy calculation}

Both the CVM and MCS require appropriate modelling of atomic interactions in order to calculate total energy change in the systems. Lennard-Jones pair potentials were employed in order to simplify the numerical simulations. The Lennard-Jones pair potentials $e_{i i}(r)$ are described by:

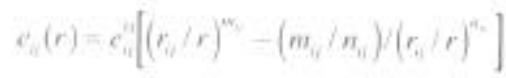

where $r_{i}$ is the interatomic distance between atomic species $f$ and $j$ for which $\epsilon_{0}(r)$ reaches is minimum value i.e. $\epsilon_{i n}(r)=-e_{0.0}^{0}$.

It has been shown by Sanchez of af.(14) that the best values for the exponents $n_{i}, m_{i, j}$ of the attractive and repulsive potentials of metals are 4 and 8 . respectively. Detaik of the method for determining pair potential parameters and the procedure for the CVM are described in previous papers $(12)$,

\section{Algorithm for MCS dati icquisition}

Fot MCS, the initial sructure may be generated by randomly assigning atoms to lattice sites. The kinetics of ordering may be controlled by the direct exchange of a randomly selected atom with one of is neighbouring atoms (Kawasiki dynamics(5)). The protability $W$ that an exchange trial is accepted is given by.

$$
W=\exp (-\Delta H / k T) / 1+\exp (-\Delta H / k T)]
$$

where $\Delta H$ is the change in energy associated with the exchange of the atoms, $k$ is the Boltzmann's constant and $T$ is the absolute tempenature. $\Delta H$ is calculated by taking account of contributions from only the first nearest neighbour atoms.

The simulations were performed on a $16 \times 16 \times 16$ unit cell mesh (16,384 atoms) mainly for visualisation, and on a $32 \times 32 \times 32$ unil cell mesh (131,072 atoms) mainly for determination of the $\gamma$ and $\gamma$ phase compositions and for the investigation of the site occupancy behaviour of alloying elements in the $\gamma^{\prime}$ phase. Periodic boundary conditions were employed. It should be noted that all the lattice positions in the system were fixed: i.e, no relaxation effects were considered in the calculation. A Hewlett Packard Model $712 / 80$ workstation system was used for the calculations. The simulations were carried out until the atomic configurations were regarded at equilibrium (typically 20,000 Monte Carlo steps): the total enthalpy change of the system proved to be negligibly small after 20,000 Monte Carlo steps,

Fig. I shows a 3-dimensional representation of the atomic arrangement of solute atoms in TMS-71, obtained by MCS after 20,000 Monte Carlo steps. Here, the 22 nd atomic layer of the system is highlighted. The distribution of atoms in the system may be thus described. Fig. 2 shows the 2-dimensional arrangement of atoms on the 22 nd to the 25 th layers, nomal to the $<100>$ direction. The atom species in TMS-71 may be identified by their size and brightness. Since pure $\mathrm{Ni}$ layers and $\mathrm{Ni}-\mathrm{Al}$ mixed layers must appear in turn along $\langle 100\rangle$ for pure fully-ordered $\mathrm{Ni}_{3} \mathrm{Al}$, the atomic locations on the $(100)$ layers in the $\gamma^{\prime}$ phase for multicomponent $\mathrm{Ni}$-base superalloys will be represented as shown in Fig. 3. From MCS, it is thus possible to identify the ordered $\gamma$ phase, as indicated by arrows in Fig. 2. the atomic configurations of which ate almost identical to the expected representation (Fig. 3). From Fig. 2, the site occupancy of alloying elements may also be understood.

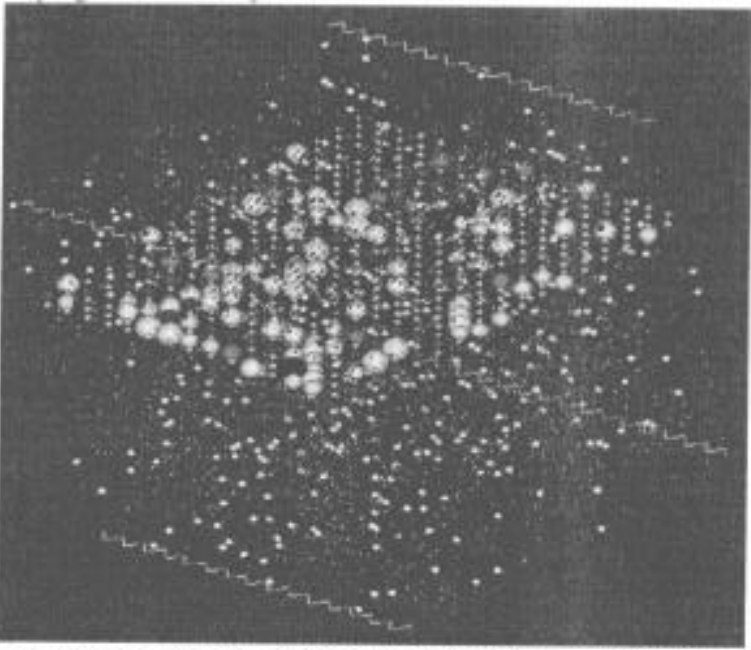

Figure 1. The 3-dimensional representation of the alomic amangensent of wolute atoms in TMS.71, oblained by MCS afler 20,000 Mtonte Carlo steps 


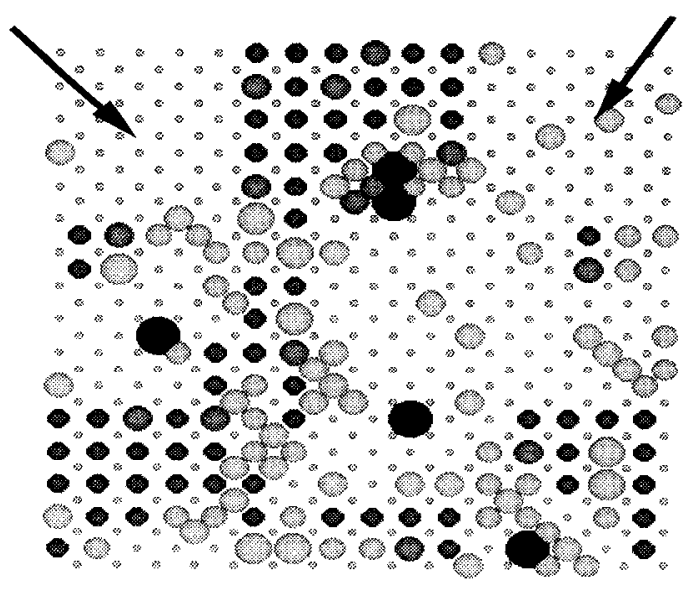

22

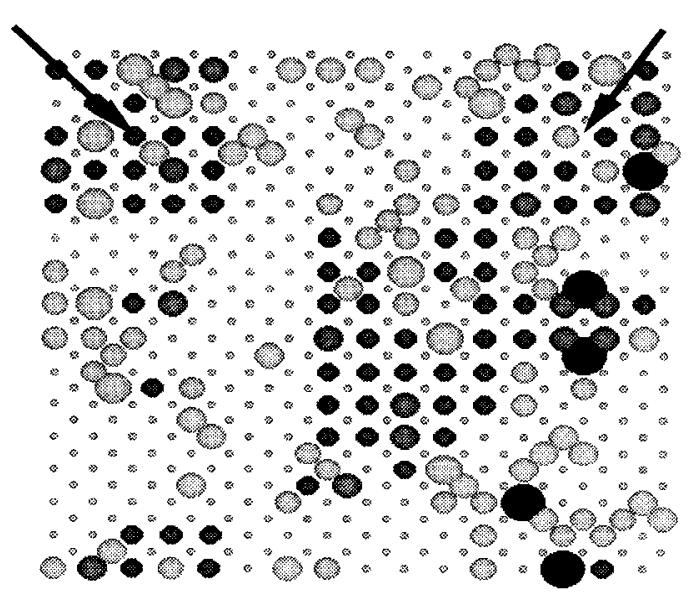

23

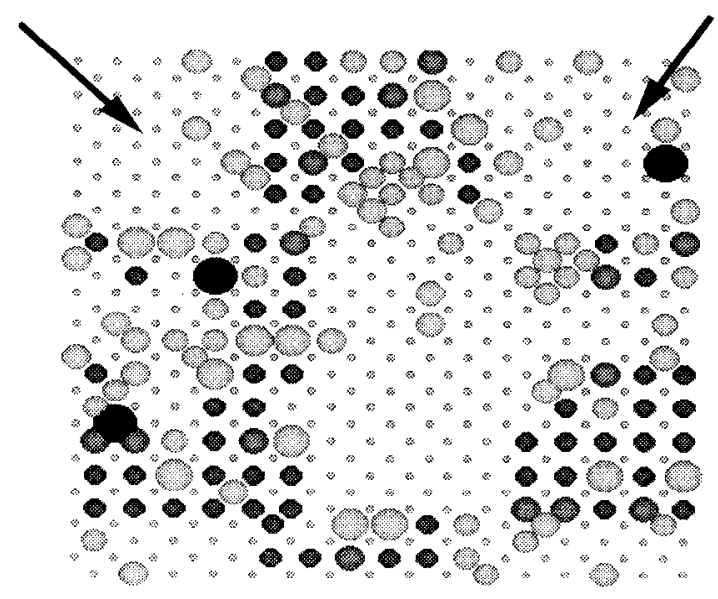

24

$\mathrm{Ni} A \mathrm{~A}$

Wra

MO

1... $\mathrm{Ta}$

Figure 2. The 2-dimensional arrangement of atoms on the 22 nd to the 25 th layers, normal to the $<100>$ direction.

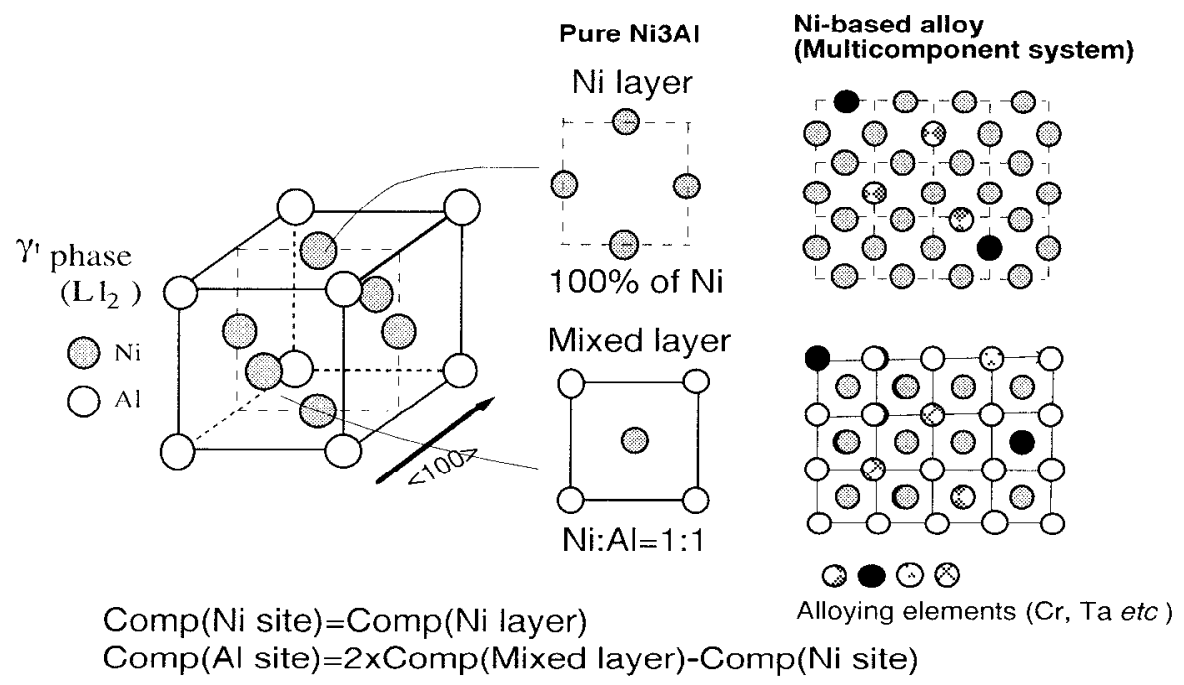

Figure 3. The expected atomic configurations of (100) layers in the $\gamma$ phase. 
Determination of $\gamma$ and $\gamma^{\prime}$ phase compositions and site occupancy behaviour in the $\gamma$ phase.

Although the $\gamma$ and $\gamma^{\prime}$ phases may be visualised from MCS, the deternination of the $\gamma$ and $\gamma^{\prime}$ phase compositions requires analysis based on the identification of neighbouring atoms. Consider a selected atom: if its 1st neighbouring atoms are mainly $\mathrm{Ni}$, and the second neighbouring atoms are mainly $\mathrm{Al}$, the selected atom is deemed to occupy the Al site in the $\gamma^{\prime}$ lattice. Fig. 4 shows the flow chart for the first step of analysis. Atoms which remain unidentified after step 1 are further analysed. Finally, $\gamma^{\prime}$ regions are determined by utilizing a cluster analysis method(16). Since all the atoms are positioned in either the $\mathrm{Al}$ site or Ni site when determining the $\gamma^{\prime}$ regions, site substitution behaviour is also determined. Note that in this study, regions which are not assigned as $\gamma^{\prime}$ regions are regarded as the $\gamma$ phase.

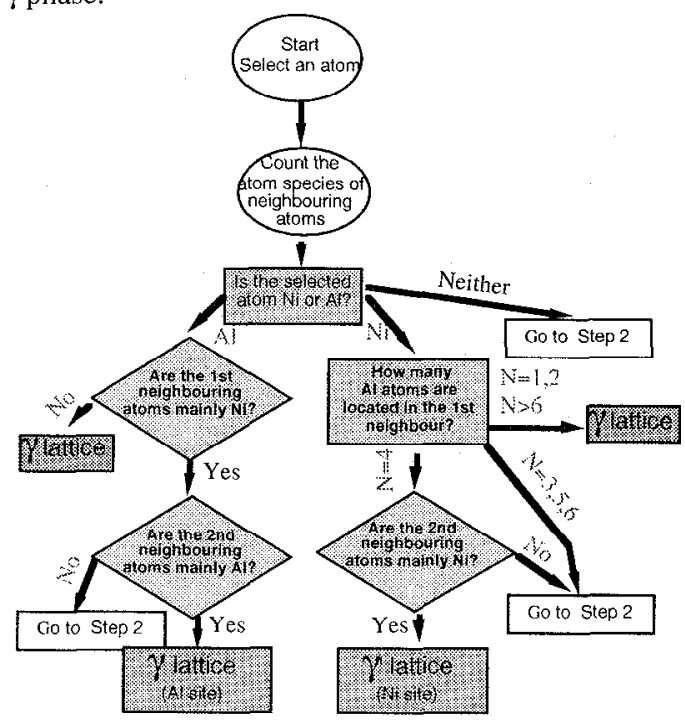

Figure 4 . The flow chart for the first step of analysis.

Results and Discussion

\section{$\gamma$ and $\gamma^{\prime}$ phase compositions}

The experimentally-determined $\gamma$ and $\gamma^{\prime}$ phase compositions obtained by APFIM were compared with data estimated by CVM and MCS. Fig. 5 shows a typical composition analysis for TMS-71; compositions estimated by the ADP are also shown. Excellent agreement is obtained, particularly for the $\gamma^{\prime}$ phase.

In order to investigate the partitioning behaviour of an alloying element ' $i$ ' into the $\gamma$ or $\gamma$ ' phlase, a partitioning parameter $k_{i}$ is defined as:

$$
k_{i}=c_{i \gamma} / c_{i \gamma}
$$

where $c_{i \gamma}$ and $c_{i \gamma^{\prime}}$ are the concentrations of an alloying element ' $i$ ' in the $\gamma$ and $\gamma^{\prime}$ phases, respectively. Thus, for example, when the element ' $i$ ' has a tendency to partition into the $\gamma$ phase. Fig. 6 summarises the partitioning behaviour of alloying elements for
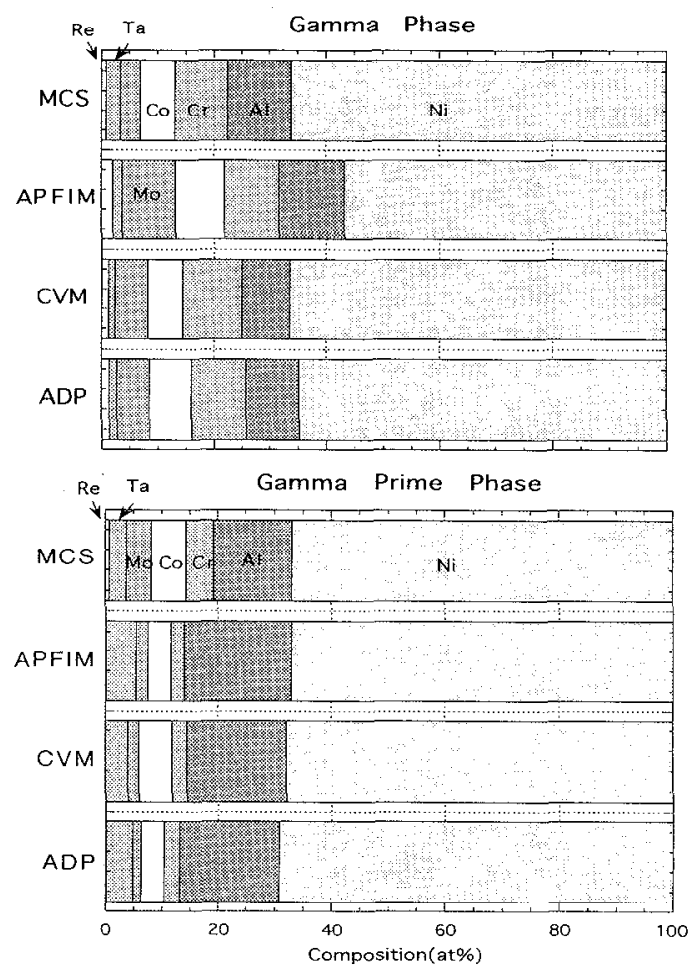

Figure 5. Compsition analysis for TMS-71.

(a) CMSX2

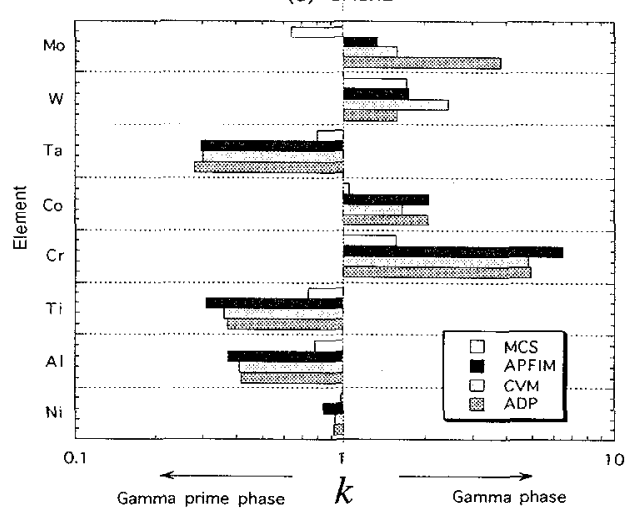

(b) $\mathrm{CMSX} 4$

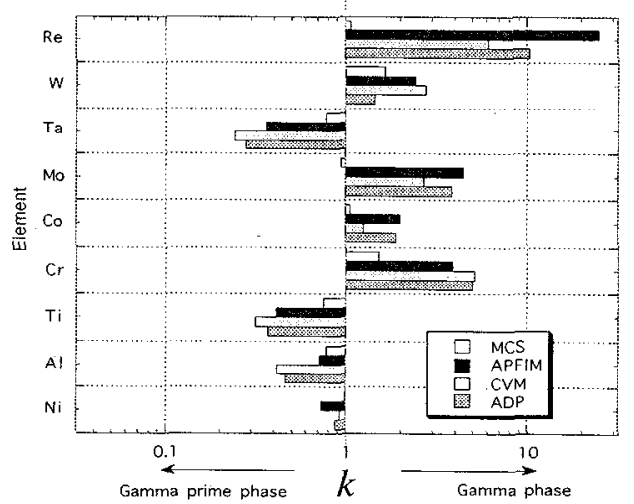

Figure 6. Partitioning behaviour of alloying elements. 
(c) $\mathrm{MC2}$

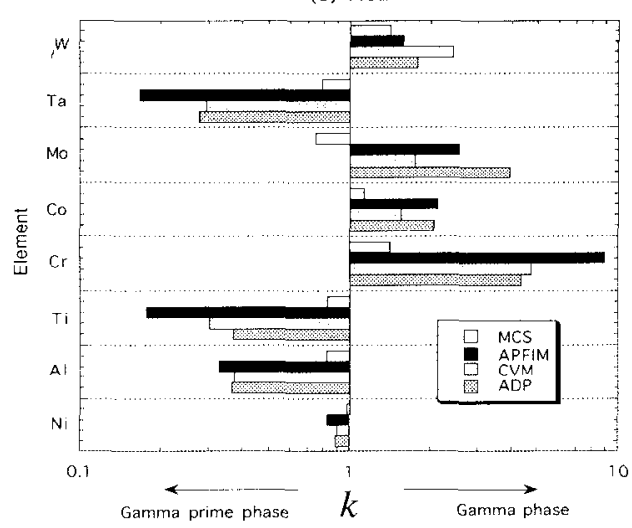

(d) TMS-G3

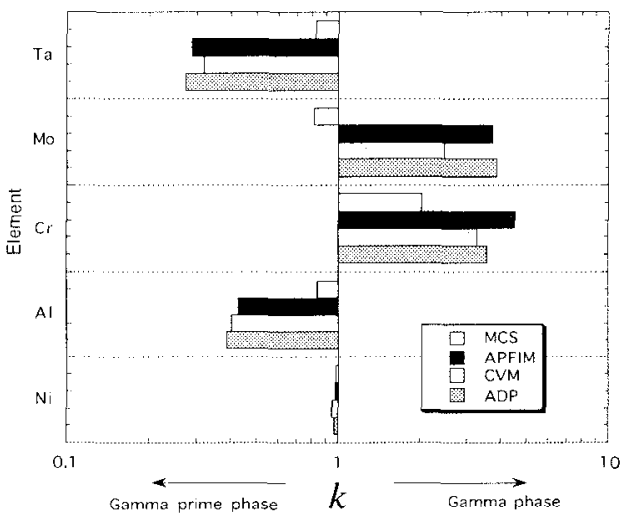

(e) TMS-71

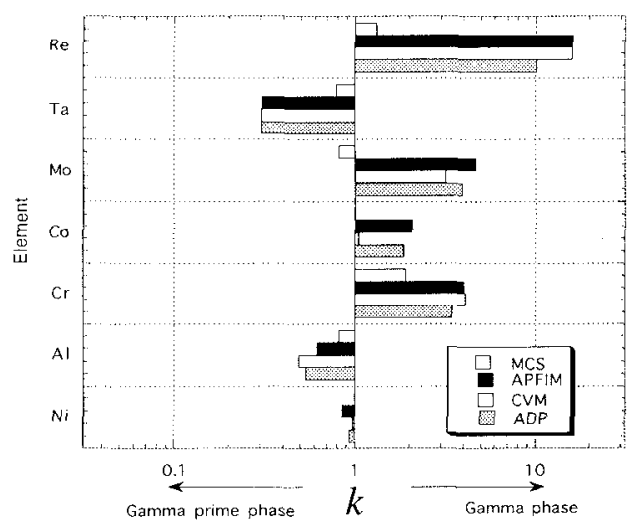

Figure 6. continued.

(a) CMSX2; (b) CMSX4; (c) MC2; (d) TMS-63 and (e) TMS-71 as a function of $k_{i}$. Hf in CMSX4 is omitted in this comparison because of its low solute content. Good agreement is shown except for the partitioning behaviour of Mo, obtained by MCS. It is also found that the partitioning tendencies predicted by MCS are not as pronounced as those obtained by other methods.

Further composition analysis revealed that $\mathrm{Ti}$ and $\mathrm{Ta}$ preferentially partition into the $\gamma$ phase while $\mathrm{Co}, \mathrm{Cr}, \mathrm{W}$ and $\mathrm{Re}$ have preference to partition into the $\gamma$ phase. CVM, ADP and APFIM analysis showed that Mo tends to partition into the $\gamma$ phase.

\section{Site occupancy of alloying clements in the $\gamma^{\prime}$ phase}

It is important to investigate the site occupancy of alloying elements in the $\gamma$ phase since whether the alloying element prefers the $\mathrm{Ni}$ sites or the $\mathrm{Al}$ sites dramatically alters the $\gamma^{\prime}$ volume fraction. Fig. 7 illustrates a simplified ternary phase diagram ${ }^{(8)}$ for a Ni-Al-M system $(\mathrm{M}=\mathrm{Ti}, \mathrm{Cr}$, Co, Mo, Ta etc.). It is well known that alloying elements which prefer the Al sites have the $\gamma^{\prime} / \gamma+\gamma^{\prime}$ phase boundary along the line $\mathrm{AB}$. On the other hand, alloying elements which prefer the $\mathrm{Ni}$ sites have the AC phase boundary. Therefore, from this figure, it may be seen that alloy $\mathrm{X}$ whose composition is pointed as - may be in the $\gamma^{\prime}$ single phase when the alloying element substitutes for the Al site, whereas it may be almost $50 \% \gamma+50 \% \gamma^{\prime}$ two-phase structure when the alloying element substitutes for the $\mathrm{Ni}$ site. Hence, alloying elements which substitute for the Al site increase the $\gamma^{\prime}$ volume fraction. Understanding the substitution behaviour of solute atoms in the $\gamma$ phase is thus essential in order to estimate the $\gamma^{\prime}$ volume fraction, which is one of the main factors in determining the mechanical properties of $\mathrm{Ni}$-base superalloys. In the case of a multicomponent system, however, the site preference of an alloying element might differ from that in a ternary system, due to the 'site competition' of other solute elements. Accordingly, it is difficult to determine the site occupancy in a multicomponent system by analysis methods such as Mössbauer spectroscopy(17). Layer-bylayer analysis employed in APFIM enables experimental determination of the site occupancies of alloying elements in multicomponent alloys. The detailed experimental procedure for layer-by layer analysis of multicomponent alloys has been reported previously $(11,12)$

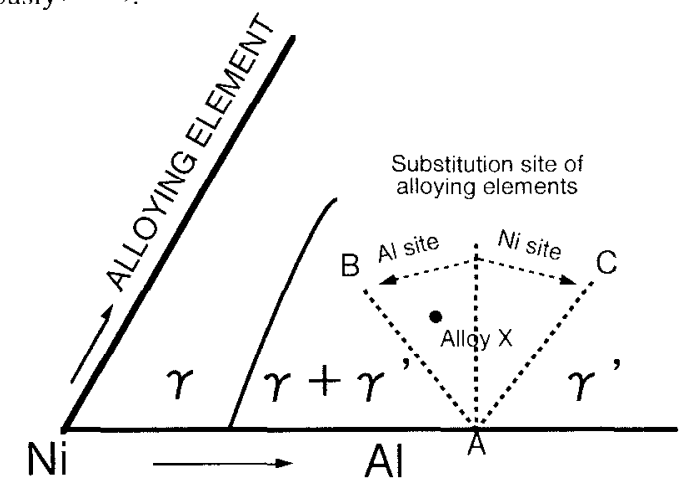

Figure 7. A simplified ternary phase diagram ${ }^{(8)}$.

Fig. 8 shows the occupancy probabilities of alloying elements on (a) Al sites and (b) Ni sites for TMS-63, estimated by MCS and CVM, and experimentally determined by APFIM. Good agreement is obtained especially for the Ni site.

In order to determine the sitc occupation bchaviour, a parameter $s_{i}$ may be defined:

$$
s_{i}=p_{i A} / c_{i \gamma^{\prime}}
$$

where $p_{i \wedge}$ and $c_{i \gamma^{\prime}}$ are the occupancy probabilities for the $\mathrm{Al}$ site and concentration in the $\gamma$ phase of an alloying element ' $i$, respectively. 
(a) Al site

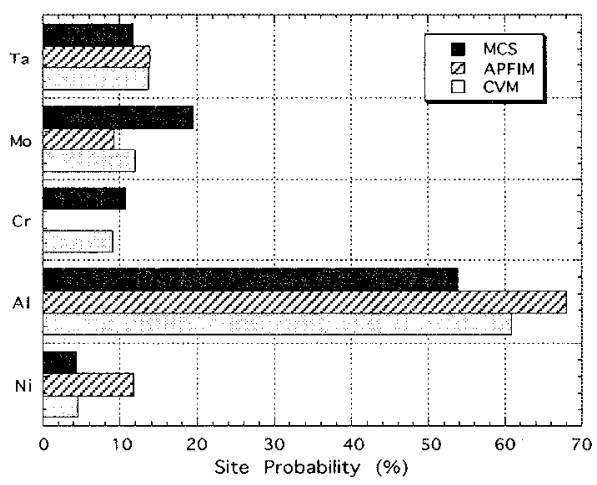

(b) $\mathrm{Ni}$ site

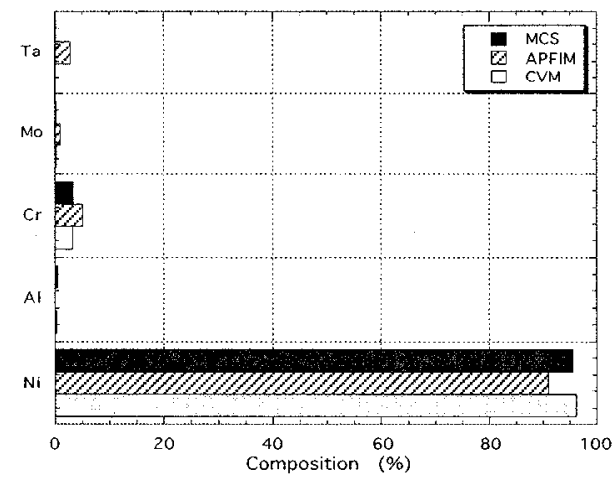

Figure 8. the occupancy probabilities of alloying elements on (a) $\mathrm{Al}$ sites and (b) Ni sites for TMS-63.

Since the number of $\mathrm{Ni}$ sites is three times that of $\mathrm{Al}$ sites for $\mathrm{Ni}_{3} \mathrm{Al}$, $s_{i}=1,2$ and 4 correspond to the occupancy behaviour of an alloying element ' $i$ ' for the cases in which atoms are randomly distributed in the two sites, an equal number of atoms enter the two lattices and all atoms substitute for the $\mathrm{Al}$ sites, respectively. Fig. 9 summarises the site occupancy behaviour of alloying elements in (a) CMSX2; (b) CMSX4; (c) MC2; (d) TMS-63 and (e) TMS-71. Here, Mo in CMSX2 and Hf in CMSX4 are omitted because of the experimental uncertainties ${ }^{(11,12)}$. In addition, APFIM analysis of TMS-71 has not yet been conducted. Generally, good agreement is observed except for some discrepancies between estimated and experimental data in the cases of $\mathrm{Co}$ and $\mathrm{Ta}$, and estimated site substitution behaviour of W obtained by MCS and CVM.

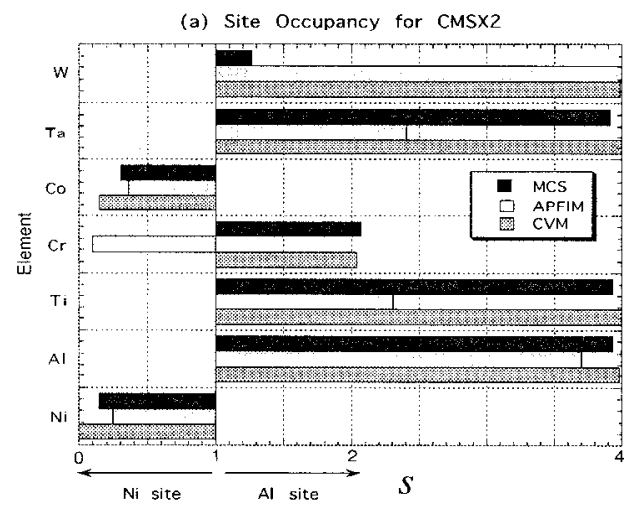

(b) Site Occupancy for CMSX4

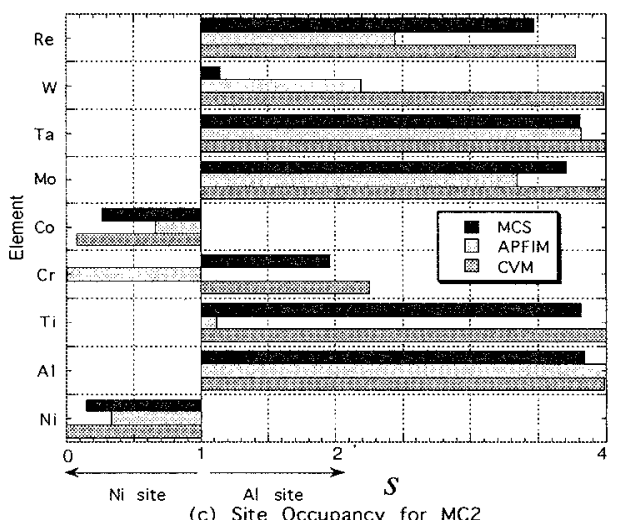

(c) Site Dccupancy for MC2

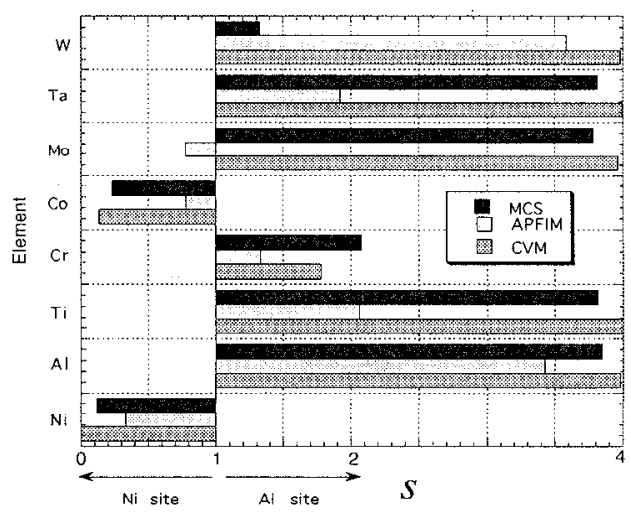

(d) Site Occupancy for TMS-63

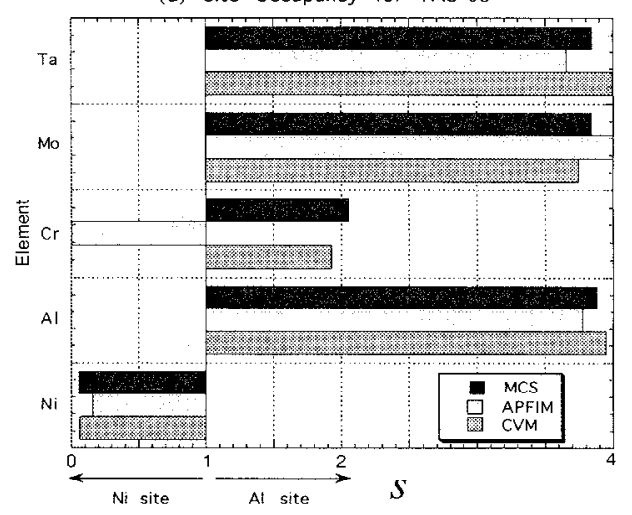

(e) Site Occuapncy for TMS-71

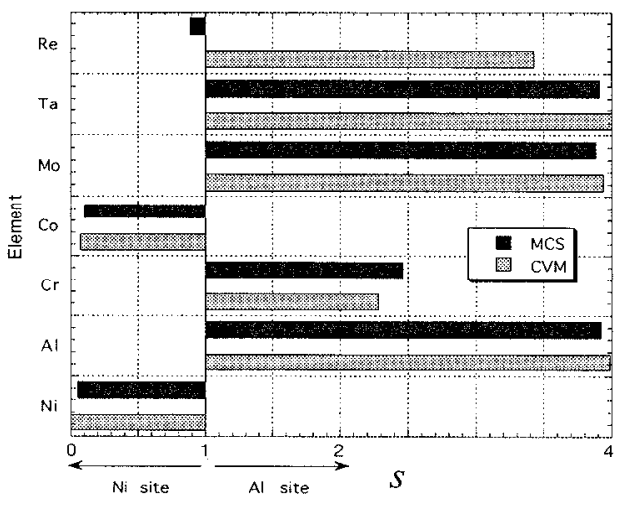

Figure 9. The site occupancy behaviour of alloying elements in $5 \mathrm{Ni}-$ base single crystal superalloys. 
Site occupancy determination for other alloys revealed that generally Mo, Ta, W, Re and Ti tend to occupy the Al sites whilst Co has a preference for the $\mathrm{Ni}$ sites. The site occupancy of $\mathrm{Cr}$ is composition dependent .

From the investigations of phase compositions and site occupancy of alloying elements in the $\gamma^{\prime}$ phase, two types of discrepancies have been found: (1) the discrepancy between experimental analysis and numerical simulations, such as the site occupancy of $\mathrm{Cr}$ in the $\gamma^{\prime}$ phase, and (2) the discrepancy between CVM and MCS, such as partitioning behaviour of alloying elements (typically $\mathrm{Mo}$ ) and the site occupancy of Re in the $\gamma^{\prime}$ phase for TMS-71 and the site occupancy of $W$ in the $\gamma$ phase for all the other alloys. Discrepancy (1) may be attributed to the determination of potential parameters for Cr. Further investigation of the experimental conditions should also be conducted.
Discrepancy (2) is caused by the difficulty in distinguishing between the $\gamma$ and $\gamma^{\prime}$ phases from MCS. As discussed above, the MCS were carried out using fixed lattice positions and identical pair potentials in both the $\gamma$ and $\gamma^{\prime}$ phases. In addition, the number of atoms used for the calculation may not be large enough to describe the atomic configurations of the two phases for multicomponent alloys. Fig. 10 shows an example of the two dimensional atomic configurations on a (100) layer for TMS -71 obtained by MCS for the $32 \times 32 \times 32$ unit cell system. Here, some Mo-Re clusters with 3-8 atom numbers are indicated by the arrows. Since the pair potential between Mo and Rc is very binding, Mo-Re atom pairs easily form in TMS-71 during MCS. W atoms behave similarly to Re for all the other alloys: e.g. Mo-W clusters are formed during calculations. These clusters frequently appear in the vicinity of $\gamma / \gamma$ interfaces or even embedded in $\gamma$ precipitates.

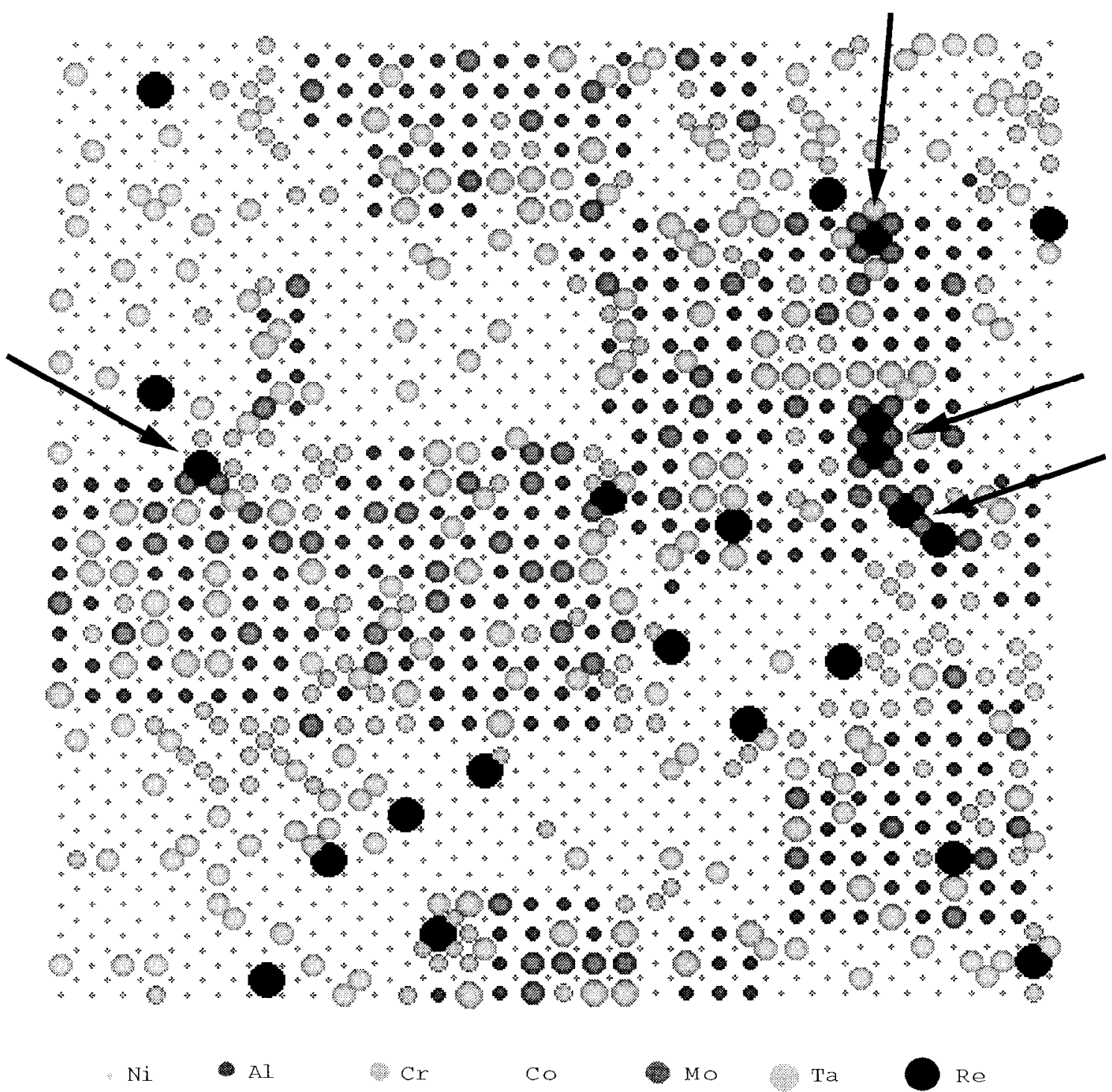

Figure 10. An cxample of the 1 wo dimensional atonic configurations on a (100) layer for TMS-71 obtained by MCS for the $32 \times 32 \times 32$ unit cell system in which some Mo-Re clusters with 3-8 atom numbers are indicated by the arrows. 
Superalloys containing large amount of solute elements tend to form a topologically close-packed (TCP) phase such as the $\mu$ phase, which has very high concentration of $\mathrm{W}$ and Mo. For example, it has been reported for $\mathrm{MC2} 2^{(18)}$ that the $\mu$ phase precipitates from the $\gamma$ matrix, preferentially in the region where the $\mathrm{W}$ level is maximum and that the $\mu$ phase particles are embedded in the $\gamma^{\prime}$ phase, and that the depletion of the matrix of elements $\mathrm{Cr}, \mathrm{Co}$ and Mo leads to the transformation $r \rightarrow r^{\prime}+\mu$. Our MCS predictions describe these results well if we consider Mo-W clusters to be the $\mu$ phase. Alternatively, the clusters might show the composition fluctuation in the $\gamma$ phase at which precipitation of the $\mu$ phase might occur. Due to the small number of atoms which forms a cluster, the analysis algorithm regards these clusters ( $\mathrm{Re}, \mathrm{Mo}$ ) as parts of the $\gamma^{\prime}$ phase, resulting in the fact that additional Mo atoms appear to be positioned in the $\mathrm{Al}$ sites in the $\gamma^{\prime}$ phase, and the neighbouring atoms regarded to be positioned in the $\mathrm{Ni}$ sites in the $\gamma^{\prime}$ phase, These results may cause the discrepancies between CVM and MCS. Employing a super computer facility, which accepts the increased system size for calculation than a conventional workstation system does, may be of help to overcome some of these limitations. The authors are also examining a modified MCS algorithm employing a local relaxation parameter ${ }^{(19)}$. It should be noted, however, that semi-quantitative agreement for compositional analysis has been obtained by MCS.

In this study, it has been shown that elements such as Mo, W and Re have a tendency to partition to the $\gamma$ phase and occupy the Al site in the $\gamma$ phase. These alloying clements therefore cause the lattice misfit between the $\gamma$ and $\gamma^{\prime}$ phases to tend towards a negative direction ${ }^{(6,9)}$ and thus, enhance the creep resistance ${ }^{(5,7)}$. However, it should be noted that the addition of these elements will increase the $\gamma^{t}$ volume fraction since they tend to substitute for the Al site. It is thus important to determine the optimum solute content in order to design alloys having superior high-temperature capabilities.

\section{Chemistry change at $\gamma / \gamma^{\prime}$ interfaces}

The composition changes at $\gamma / \gamma^{\prime}$ interfaces may be determined by APFIM analysis, since the analysis is of atoms successively fieldevaporated from the specimen surface and chemically identified. When the cumulative number of each solute atom is plotted against the total number of detected atoms in a 'ladder diagram', the horizontal axis includes depth information and the gradient corresponds to the local composition of that alloying element. Fig. 11 shows a typical ladder diagram of the composition change at a $\gamma / \gamma^{\prime}$ interface. Note the enrichment of $\operatorname{Re}$ in the $\gamma$ phase in the vicinity of the interface.

This ladder diagram may be simulated using MCS by analysing a cylinder along the $\langle 100\rangle$ direction (containing, for example, 40 atoms per atomic layer). Fig. 12 shows such a ladder diagram across a $\gamma / \gamma^{\prime}$ interface, similar to Fig. 11 except for that the number of atoms 'detected' is smaller by a factor of 10 .

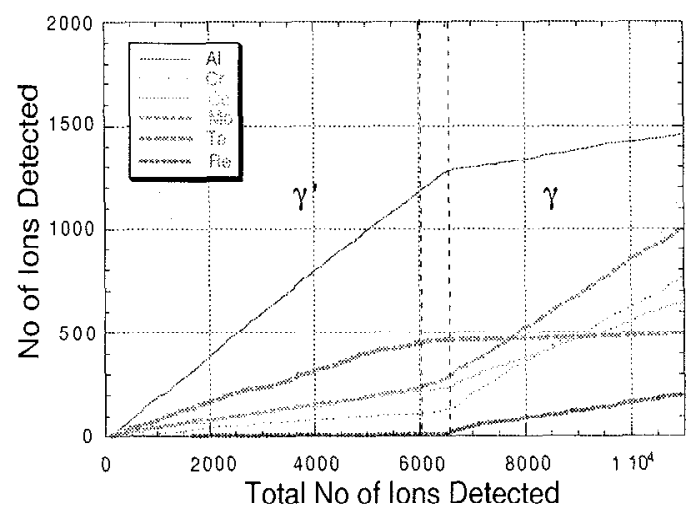

Figure 11. A typical ladder diagram of the composition change at a $\gamma /$ $\gamma$ interface.

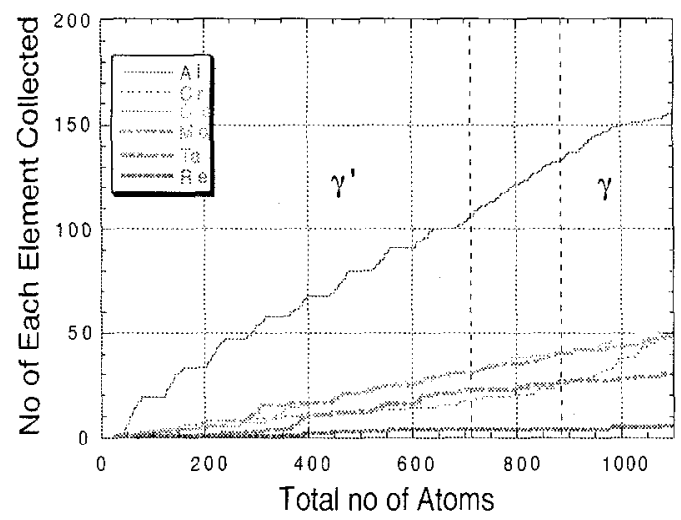

Figure 12. A 'virtual' ladder diagram obtained by the MCS.

When $\mathrm{Al}$ atoms are plotted in a ladder diagram, pure Ni-layers appear as horizontal lines whereas mixed $\mathrm{Ni} / \mathrm{Al}$ layers appear as inclined lines in the ordered $\gamma$ region. It is thus clear that the left side in Fig. 12 represents the ordered $\gamma^{\prime}$ phase and the right represents the $\gamma$ phase. The composition change between the $\gamma$ and $\gamma$ phases may also be observed for $\mathrm{Co}, \mathrm{Cr}$ and $\mathrm{Ta}$. As discussed in the previous section, however, the composition change between the $\gamma$ and $\gamma$ phases in Fig. 12 is not as clear as that experimentally obtained by APFIM. It is again suggested that further investigation such as layer by. layer analysis using APFIM, MCS with a much larger system with local relaxation factor considered etc. will be of help in obtaining more detailed comparisons between the numerical simulations and the experimental results. 


\section{Summary}

The $\gamma$ and $\gamma^{\prime}$ phase compositions and the site occupancy of alloying elements in the $\gamma$ phase were investigated by MCS. It was found that the estimates obtained by MCS are in good agreement with experimental results obtained by APFIM and CVM predictions. For the multicomponent system, alloying elements may be classified into threc groups: (1) clements which prefcrentially partition into the $\gamma^{\prime}$ phase and substitute for the $\mathrm{Al}$ sites in the $\gamma^{\prime}$ phase, such as $\mathrm{Ti}$ and Ta; (2) elements which preferentially partition into the $\gamma$ phase and substitute for the $\mathrm{Ni}$ sites in the $\gamma^{\prime}$ phase and (3) elements which generally partition into the $\gamma$ phase and substitute for the Al sites in the $\gamma^{\prime}$ phase, such as $\mathrm{Re}$, Mo and $\mathrm{W}$, dependent upon alloy composition. The simulation of APFIM analysis has been demonstrated by MCS, by use of a 'virtual' ladder diagram. It is found that ordered and disordered regions in the system are clearly identified by this representation.

The employment of a supercomputer which has much higher performance than workstations will allow an increase in the size of the calculated system and the introduction of a local relaxation factor will allow the investigation of some of the discrepancies between MCS and APFIM, or MCS and CVM, such as the partitioning behaviour of Mo and the substitution behaviour of $\mathrm{W}$ and Re.

\section{Acknowledgment}

Part of this work was carried out under the auspices of the U.K.Japan Cooperative Science Program, sponsored by The Royal Society and The Japan Society of the Promotion of Science. The authours are also very grateful to Prof. M.Enomoto at Ibaraki University and Dr. H.G.Read at NRIM for many useful suggestions.

\section{References}

1. M.Enomoto and H.Harada, "Analysis of $\gamma / \gamma$ Equilibrium in NiAl-X Alloys by the Cluster Variation Method with the Lennard-Jones Potential," Metall. Trans., 20A(1989), 649-664.

2. M.Enomoto, H.Harada and M.Yamazaki, "Calculation of $\gamma / \gamma$ Equilibrium Phase Compositions in Nickel-base Superalloys by Cluster Variation Mcthod," CALPHAD, 15(1991) 143-158.

3. Y.Saito, submitted to Mater. Sci. Eng. A.

4. P.Caron and T.Khan, "Improvement of Creep Strength in a

Nickel-base Single-crystal Superalloy by Heat Treatment," Mat. Sci. Eng. 61(1983), 173-184

5. D.J.Frasier et al., "Process and Alloy Optimization for CMSX-4 Superalloy Single Crystal Airfoils," Proceedings of the conference "High Temperature Materials for Power Engineering 1990," Liège, Belgium (1990), 1281-1300.

6. H. Harada et al., "Design of High Specific-strength Nickel-base Single Crystal Superalloys," Proceedings of the conference "High Temperature Materials for Power Engineering $1990_{2}$ "Liège, Belgium (1990), 1319-1328.

7 H. Harada et al, "Computer Analysis on Microstructure and Property of Nickel-base Single Crystal Superalloys," Proceedings of the 5th International Conference on Creep and Fracture of

Engineering Materials and Structures, Swansea, U.K,.(1993) 255 261.

8 H.Murakami, P.J.Warren and H.Harada, "Atom-probe

Microanalyses of Some Ni-base Single Crystal Superalloys,"
Proceedings of the 3rd International Charles Parsons Turbine Conference "Materials Engineering in Turbines and Compressors", New Castle, U.K., (1995) 343-350.

9 T.Yokokawa et al., "Towards an Intelligent Computer Program for the Design of Ni-base Superalloys," Proceedings of the 5th International Conference on Creep and Fracture of Engineering Materials and Structures, Swansea, U.K...(1993) 245-254.

10 H.Harada et al., "Atom-probe Microanalysis of a Nickel-base Single Crystal Superalloy," Appl. Surf. Sci., 67(1993) 299-304.

11 H.Murakami, H.Harada and H.K.D.H.Bhadeshia, "The Location of Atoms in Re- and V-containing Multicomponent Nickelbase Single-crystal superalloys," Appl. Surf. Sci., 76/77(1994)177183.

12 D.Blavette and A.Bostel, "Phase Composition and Long Range Order in $\gamma$ phase of a Nickel Base single Crystal Superalloy CMSX2: An Atom Probe Study," Acta Metall., 32(1984) 811-817. 13 S.Duval et al., "Phase Composition and Chemical Order in the Single Crystal Nickel Base Superalloy MC2," Acta Metall. Mater., 42(1994) 185-194.

14 J.M.Sanchez et al., "Modeling of $\gamma / \gamma$ ' Phase Equilibrium in the Nickel-aluminum System," Acta Metall., 33(1984) 1519-25.

15 K.Kawasaki, "Diffusion Constants near the Critical Point for Time-Dependent Ising Models. I," Physical review, 145(1966) 224230.

16 S.Sakamoto and F.Yonezewa, "The Cluster Analysis Method using Computers and its Applications," Kotaibutsuri, 24(1989), 219 226 (in Japanese).

17 J.R.Nicholls and Rees D.Rawlings, "A Mössbauer Effect Study of Ni3Al with Iron Additions," Acta Metallurgica, 25(1976), 187 194.

18 M.Pessah, P.Caron and T.Khan, "Effect of $\mu$ Phase on the Mechanical Properties of a Nickel-base Single Crystal Superalloy," Proceedings of Superalloys 1992, Seven Springs, U.S.A., (1992), $567-576$

19 Y.Saito H.Murakami and H.Harada, submitted to the proceedings for the International Workshop on Computer Modelling and Simulation for Materials Design, Tsukuba Science City, Japan, (1996). 